\title{
Discovery and Spectroscopy of Dark Matter and Dark Sectors with Microscopic Black Holes at Next Generation Colliders
}

\author{
Ningqiang Song $\odot^{*}$ and Aaron C. Vincent $\odot^{\dagger}$ \\ Department of Physics, Engineering Physics and Astronomy, Queen's University, Kingston, Ontario K7L 3N6, Canada \\ Arthur B. McDonald Canadian Astroparticle Physics Research Institute, Kingston, Ontario K7L 3N6, Canada \\ Perimeter Institute for Theoretical Physics, Waterloo, Ontario N2L 2Y5, Canada
}

(Received 25 July 2019; revised manuscript received 18 October 2019; accepted 13 January 2020; published 3 February 2020)

\begin{abstract}
If the length scale of possible extra dimensions is large enough, the effective Planck scale is lowered such that microscopic black holes could be produced in collisions of high-energy particles at colliders. These black holes evaporate through Hawking radiation of a handful of energetic particles drawn from the set of all kinematically and thermally allowed degrees of freedom, including dark matter. Here, we perform the first numerical black hole spectroscopic study of the dark sector. We find that if the next generation of colliders can produce microscopic black holes, then missing momentum signatures can reveal the existence of any new light ( $\lesssim 10 \mathrm{TeV}$ ) particle, regardless of the strength of its coupling to the standard model, even if there exists no such nongravitational coupling at all.
\end{abstract}

DOI: 10.1103/PhysRevLett.124.051801

Introduction.-Significant experimental effort is underway to uncover the nature of dark matter (DM), which makes up approximately $85 \%$ of the matter in the observable Universe [1]. Cosmological observations dictate that the DM cannot interact strongly with particles from the standard model (SM) of particle physics, and must behave on large scales like a cold, collisionless particle. There are theoretical motivations for a particle physics "portal" to the dark sector (DS) via some weak-scale interaction, which mainly have to do with the relative abundance of DM versus SM matter in the Universe [2,3]. Despite extensive experimental searches, all evidence of DM has remained in its gravitational interactions on the scale of galaxies and larger.

There exists a possibility, sometimes dubbed the "nightmare scenario," that the DM-and indeed its possible extension to the dark sector-is entirely secluded from the SM, interacting only via gravity. This is a disheartening prospect. However, we will show that in the presence of large enough extra dimensions to bring the Planck scale down near the electroweak scale, all hope is not lost.

Large extra dimensions (LEDs) have been proposed for a multitude of theoretical and phenomenological reasons, and $\mathrm{TeV}$ scale quantum gravity in particular offers a compelling solution to the hierarchy problem [4-9] that exists between the Planck and electroweak scales. In such

Published by the American Physical Society under the terms of the Creative Commons Attribution 4.0 International license. Further distribution of this work must maintain attribution to the author(s) and the published article's title, journal citation, and DOI. Funded by SCOAP ${ }^{3}$. scenarios, the $\mathrm{SM}$ is confined to a $3+1$ dimensional brane, and only gravity may propagate in the full $D=4+n$ dimensional bulk. Depending on the number, size, and geometry of the LEDs, the true Planck scale $M_{\star}$ can be much lower than the effective Planck scale $M_{\mathrm{Pl}} \sim$ $10^{18} \mathrm{GeV}$ seen on our brane. These scenarios have been tested extensively in terms of gravitational force [10], supernova and neutron star cooling [11,12], the metastability of the Higgs vacuum [13], and at colliders [14,15], as we shall scrutinize below. If only one LED exists, this solution leads to modifications of gravity on Solar System scales [4]. However, two or more LEDs remain allowed. Current constraints limit the scale $M_{\star}$ to be above about 3-25 TeV [10-15].

A profound consequence of a low Planck scale is the possibility of creating microscopic black holes (BHs) in high-energy collisions. Indeed, the hoop conjecture $[16,17]$ implies that as long as the impact parameter $b$ between two colliding particles is smaller than twice the horizon radius $r_{h}$ of a $\mathrm{BH}$ with mass $M_{\mathbf{0}}=E_{\mathrm{c} . \mathrm{m} \text {. }}$, the center-of-mass energy, then a BH will form. The phenomenology of BHs at colliders is a rich and well-studied topic [18-29], and has mainly focused on the prospects and methods of detecting such objects, as they rapidly evaporate via Hawking radiation [30].

$\mathrm{BH}$ evaporation is effectively instantaneous, and because of the very small masses, the BH will only emit a few ( 6-20, depending on initial BH mass) particles in its decay. Crucially, decay products are a subset of every bulk degree of freedom (d.o.f.), drawn from a thermal distribution at the Hawking temperature $T_{H}$, independently of any particle physics. By carefully measuring the energy and momentum output of a large enough sample of decaying 
$\mathrm{BHs}$, one in principle has full access to any sequestered DS. In practice, missing energy and momentum during a decay process allow us to count the number of invisible d.o.f., including neutrinos, gravitons, and dark matter, allowing for a discovery of the DS particles. We also note that the aforementioned collider bounds on LEDs rely on the assumption that BHs only decay to SM matter and gravitions. Evaporation into a large DS may lead to a reconsideration of these bounds, and the Planck scale can be significantly lowered.

Two basic conditions need to be fulfilled: (i) the full $D$ dimensional Planck scale must be low enough to allow production at future colliders, and (ii) the masses of the DS particles must be lower than the $\mathrm{BH}$ mass. As long as $T_{H}$, which is typically large, remains near or above the DM mass, production is not Boltzmann suppressed. There is no lower limit to the masses that can be probed. For this study, we consider a dark sector with particle masses $m_{i} \ll M_{\star}$. Our conclusions hold in the most generic scenario in which all particles except gravitons are confined to our 3-brane, or to one that is parallel. Even if there is a dark sector that can propagate in the bulk, only the projection of its momentum onto our brane can be seen. However, emission in the bulk is kinematically suppressed because the $\mathrm{BH}$ must conserve gauge charge, and will thus be confined to our brane in most instances.

Some of the strongest limits on $\mathrm{BH}$ production come from the Large Hadron Collider, with c.m. collisions limited to $14 \mathrm{TeV}$. We thus turn to the next generation of colliders, such as the Future Circular Collider (FCC) [31] or Super Proton-Proton Collider (SPPC) [32], with a c.m. energy of $100 \mathrm{TeV}$.

We consider a simple LED scenario, with a single, tensionless brane, leaving a fuller exploration of the LED parameter space to future work. We will assume that the actual detection of such BHs is well understood, and focus on the use of BHs as probes of new particle physics. This is reasonable, as the high-multiplicity, high-transverse momentum signature of a $\mathrm{BH}$ event is very distinct from known SM processes, appearing in signal regions with almost no expected SM background (see, e.g., Refs. [33,34] and references above).

We first review the essential elements of $\mathrm{BH}$ production and evaporation at colliders. Then, we incorporate the extended DS into a full simulation of BH production and evaporation in order to carefully quantify the missing momentum signature accessible to future colliders. We end with a discussion of future directions.

$\mathrm{BH}$ production and decay at colliders.-The $\mathrm{BH}$ production cross section is [35]

$$
\sigma^{\mathrm{pp} \rightarrow \mathrm{BH}}=\int_{M_{\star}^{2} / s}^{1} d u \int_{u}^{1} \frac{d v}{v} \pi b_{\max }^{2} \sum_{i, j} f_{i}(v, Q) f_{j}(u / v, Q),
$$

where $b_{\max }=2 r_{s}^{(D)}\left(E_{\mathrm{c} . \mathrm{m} .}\right) /\left[1+(D-2)^{2} / 4\right]^{1 /(D-3)}$ is the maximum impact parameter that allows $\mathrm{BH}$ production (we work in natural units: $c=\hbar=k_{B}=1$.) $\sqrt{s}$ is the center-ofmass energy of a hadron-hadron collision, and

$$
r_{s}^{(D)}=k_{D} M_{\star}^{-1}\left(\frac{\sqrt{u s}}{M_{\star}}\right)^{1 /(D-3)}
$$

is the $D$-dimensional Schwarzschild radius of a $\mathrm{BH}$ with mass $M .=\sqrt{u s} \equiv E_{\text {c.m. }} \cdot k_{D}$ is a geometrical factor related to the number of extra dimensions. The factors of $f(v, Q)$ represents the parton distribution functions (PDF) of each constituent of the colliding hadrons, which are summed over all quark flavors and gluons. $v$ and $u / v$ are the fraction of c.m. energy carried by each colliding parton, and $Q^{2}$ is the square of the exchanged momentum four-vector. The formation of a stationary $\mathrm{BH}$ is usually associated with partial loss of its energy, momentum, and angular momentum due to the nonlinearity in the collision, which can affect the total formation cross section. We do not include this initial energy loss, as only a few numerical studies have attempted to quantify it [36]. Because the semiclassical treatment (1) must break down near $M_{\star}$, the minimum energy required to form a $\mathrm{BH}, M_{\min }$ could be different. In the absence of a detailed theory, we make the simplifying assumption $M_{\min }=M_{\star}$.

Once produced, the $\mathrm{BH}$ will evaporate via Hawking radiation, followed by what is modeled as a final "burst" as the BH mass approaches $M_{\star}$. Hawking evaporation proceeds with a temperature $T_{H}=(D-3) / 4 \pi r_{h}$ (shown in the left panel of Fig. 1, see later for detailed discussion). In the absence of angular momentum, the horizon radius $r_{h}$ is equal to $r_{s}$. There are two contributions to an effective deviation from a thermal spectrum: first, a "greybody" factor must be included to account for the distortion of the thermal spectrum as it is ultimately seen by an observer at "infinity," rather than at the horizon. Second, since $T_{H}$ is similar in magnitude to $M_{\text {. }}$, only a few particles are produced per $\mathrm{BH}$, so a continuum is not expected. Moreover, as a complete theory of gravity below $M_{\star}$ is missing, the semiclassical approach is bound to fail in the final stages of evaporation. We make the typical assumption that a minimum number of particles that conserve the energy, momentum, angular momentum, and all the gauge charges of the $\mathrm{BH}$ are emitted in a final burst when $M_{\bullet}$ nears $M_{\star}$.

Including all polarization states, the SM contains 118 d.o.f., of which 6 (neutrinos) can be considered completely invisible. A BH may also decay invisibly to gravitons, which have $D(D-3) / 2$ polarization states.

The fraction of the BH energy emitted invisibly should be approximately

$$
f_{\text {inv }}=\frac{N_{\nu}+N_{G}+N_{\mathrm{DM}}}{N_{\mathrm{vis}}+N_{\nu}+N_{G}+N_{\mathrm{DM}}},
$$



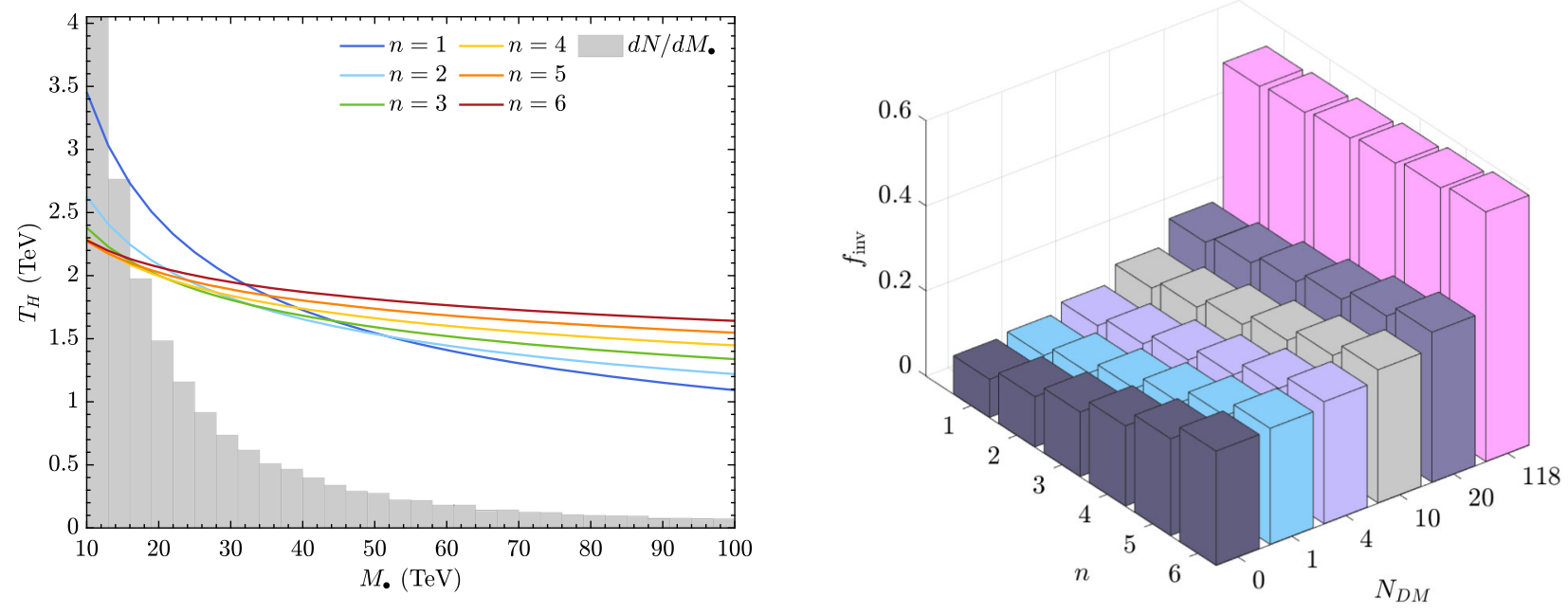

FIG. 1. Left: Lines: Hawking Temperature $T_{H}$ as a function of the microscopic BH mass, for $M_{\star}=10 \mathrm{TeV}$ in the case of $n=1$ to 6 extra dimensions. Grey bands represent the distribution of BH masses created in $100 \mathrm{TeV}$ proton-proton collisions (arbitrary units). Right: the fraction of invisible energy released from the evaporation of BHs, for different values of the number of extra dimensions $n$, and dark sector d.o.f., $N_{\mathrm{DM}}$.

where $N_{\text {vis }}, N_{\nu}, N_{G}$, and $N_{\text {DM }}$ are the respective number of d.o.f. in the visible, neutrino, graviton, and dark sectors. The fraction of invisible decay products from evaporating BHs with one extra dimension is around $9 \%$ when only SM particles are present; this rises to $12 \%$ in the presence of a single dark Dirac fermion (4 d.o.f.), a $\sim 30 \%$ increase. Figure 1 shows this fraction as a function of the number of extra dimensions $n=D-4$ and the number of dark d.o.f. $N_{\mathrm{DM}}$.

Heavy leptons, weak bosons, and hadronic jets further release some energy into neutrinos, and other charged decay products may have too low an energy to be detected. We account for the former in our full simulations, but not the latter as we are interested in the theoretical expectation.

In a hadron collider, collision occurs between two partons, as in Eq. (1), so the total energy in the interaction is not known. We thus rely on missing transverse momentum, denoted $\not \not^{\perp}$, defined as the total unbalanced momentum transverse to the collision axis. To compute missing momenta, we turn to a full numerical simulation of $\mathrm{BH}$ production, evaporation, and showering.

Numerical simulations. - Because of the small (20 or fewer) number of particles produced per $\mathrm{BH}$ evaporation, collider signatures can be highly anisotropic. To simulate this process, we first employ a modified version of the BlackMax [35,37] code. BlackMax is a Monte Carlo event generator which simulates production and evaporation of $\mathrm{BHs}$ in $p p, p \bar{p}$, and $e^{+} e^{-}$collisions. It includes known greybody factors, can account for nonzero angular momentum, and $\mathrm{BH}$ recoil in the bulk. Given a fixed proton-proton c.m. energy, BlackMax generates black holes distributed as an unintegrated Eq. (1), i.e., proportional to $d \sigma^{\mathrm{pp} \rightarrow \mathrm{BH}} / d u$.

Rather than using the built-in PYTHIA 6 interface, we pass the results of BlackMax to PYTHIA 8 [38] to account for
QCD radiation, hadronization of quark and gluon final states and decay of heavy particles. In both steps, we employ the CT14NNLO [39] PDFs, implemented with LHAPDF6 [40].

We modify the BlackMax code to add a variable number of new dark particle d.o.f. to the BH evaporation spectrum. We take each d.o.f. to obey the thermal and greybody distributions of scalar bosons. (We have verified that using Fermi-Dirac statistics and greybody factors instead does not significantly alter our conclusions.) Greybody factors for bulk emission of gravitons from rotating BHs are not available (specifically superradiance can be important for extremely rotating BHs [41]), and are thus not implemented in BlackMax. We therefore simplify our simulations to consider only nonrotating $\mathrm{BHs}$, since the emission of bulk gravitons has a more important effect on missing energy.

We simulate $\mathrm{BH}$ production and evaporation in the case of 1-6 extra dimensions. ( $n=1$ is strongly excluded, but we keep it for illustrative purposes.) We take the protonproton c.m. energy $\sqrt{s}=100 \mathrm{TeV}$, and the Planck scale $M_{\star}=10 \mathrm{TeV}$, to be compatible with current collider multijet [14] and dijet [15] searches. We also assume the minimum BH mass $M_{\min }$ is of the order of $M_{\star}$ and neglect any possible energy loss in the phase before the formation of a stationary $\mathrm{BH}$.

For each scenario, we simulate $10^{4} \mathrm{BH}$ events, assuming $0,1,4,10,20$, and 118 dark d.o.f., as a representation of possible extended dark sectors. Four d.o.f. could represent a single Dirac fermion; $\mathrm{O}(10)$ d.o.f. could include new dark forces, while 118 d.o.f. correspond to an entire "mirror" sector of the SM [42].

We assume that $\mathrm{BH}$ events can be clearly identified, and focus on what we learn from missing transverse momentum from such events. After primary particle hadronization and 


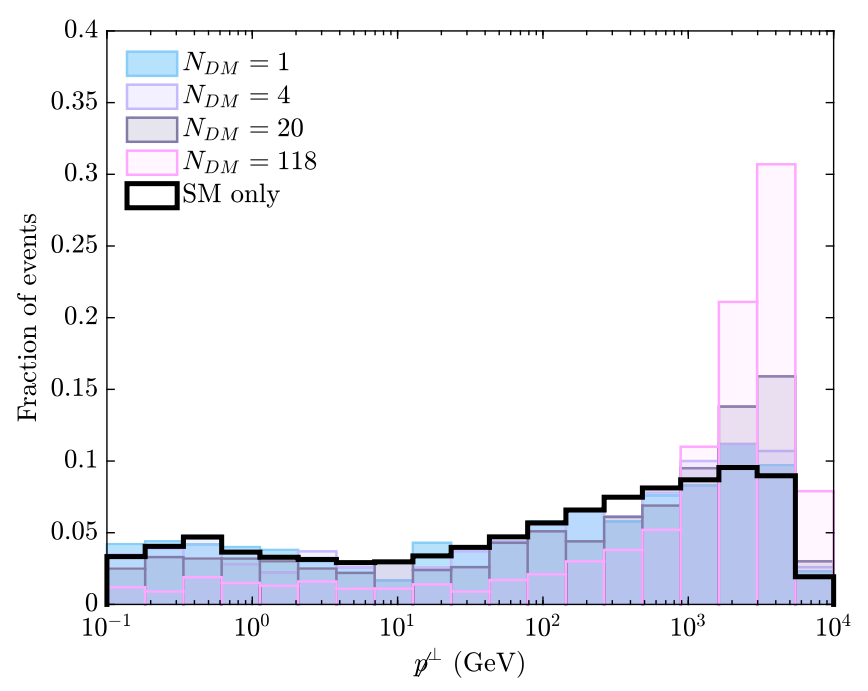

FIG. 2. Distribution of missing transverse momentum from $\mathrm{BH}$ evaporation for $n=2$ extra dimensions. In black we show the SM-only case, and in colour, the case where the dark sector contains $N_{\mathrm{DM}}=1,4,20$ and 118 new particles with masses $m_{i}<M$.. Missing momentum from SM background sources are not included here.

decay, the momenta of the remaining visible particles are added vectorially, in the plane transverse to the beam pipe. For each $\mathrm{BH}$, the magnitude of this vector corresponds by definition to $\not{ }^{\perp}$.

Figure 2 is a histogram of $\not p^{\perp}$ from a sample of simulated evaporating $\mathrm{BHs}$ in the case of two extra dimensions, when only neutrinos and gravitons are emitted (black, $N_{\mathrm{DM}}=0$ ), and for $N_{\mathrm{DM}}=1,4,20$, and 118 new d.o.f. As the number of dark d.o.f. increase, the mean $\not p^{\perp}$ rises sharply, and the distribution becomes much more peaked. This is due to the increase in primary particles that carry away a large fraction of the BH's energy. BHs decaying to small amounts of $\not p^{\perp}$, which mainly comes from the partial invisible decay of heavy SM states, become rarer. This trend does not change with the number of extra dimensions. We note that the main difference in distributions comes from the high $\not \not^{\perp}$ region, further motivating our decision to neglect low-energy cuts. As stated earlier, we are not including backgrounds from $\mathrm{SM}$ processes (i.e., when no $\mathrm{BH}$ is formed), as they are expected to be sufficiently far from the $\mathrm{BH}$ signal region.

Assuming the missing momentum for each event can be well reconstructed, we wish to know how many events must be observed to establish the existence of an extended DS. We construct reference distributions for the cases of $n=1$ to 6 extra dimensions using $10^{6}$ SM-only (i.e., $N_{\text {DM }}=0$ ) simulated events each (for a total of $\sim 150$ CPU days), which we use to compute a Kolmogorov-Smirnov (KS) $p$ value, where we have made the assumption that the number of LEDs is known. The KS test assesses how likely it is that a given data sample was drawn from the reference distribution, by comparing the maximum difference in their cumulative distribution functions, and is independent of any binning. The interpretation of the $p$ value remains the same as other methods.

Figure 3 shows the KS $p$ values, depending on the number of $\mathrm{BH}$ events recorded $N$., which we sample randomly from our simulated events. For each value of $N$. we draw 300 samples, and average the $p$ value to obtain an expected sensitivity. Small features nonetheless remain, reflecting the random sampling. We have verified that adding a 10\% random Gaussian error on the reconstructed momenta does not affect our results, and the main source of uncertainty remains statistical. The colored bands show the full range of $p$ values spanned by the six cases of $n=1-6$ extra dimensions. The thin lines show these results for each value of $n$, where $n=1$ is always the strongest-constrained (leftmost), and $n=6$ is the weakest. This is as we expect from Fig. 1. Most BHs produced are near the $10 \mathrm{TeV}$ threshold, where $T_{H}$ is highest at low $n$, leading to higherenergy particles; in higher dimensions, the extra gravitons add to the background missing energy, while the energy per DM particle is lower. For reference, $p<3 \times 10^{-7}$, equivalent to the standard $5 \sigma$ discovery threshold, is shown as a horizontal dotted line.

Depending on the number of extra dimensions, and the size of the extended dark sector, only a few hundred to a few thousand $\mathrm{BH}$ events are required to robustly determine its existence. For $N_{\mathrm{DM}}=1$, more than $10^{4}$ events are required. In the case of 4 d.o.f., a strong detection can be made with 5000 to 10000 events. Extending the DS

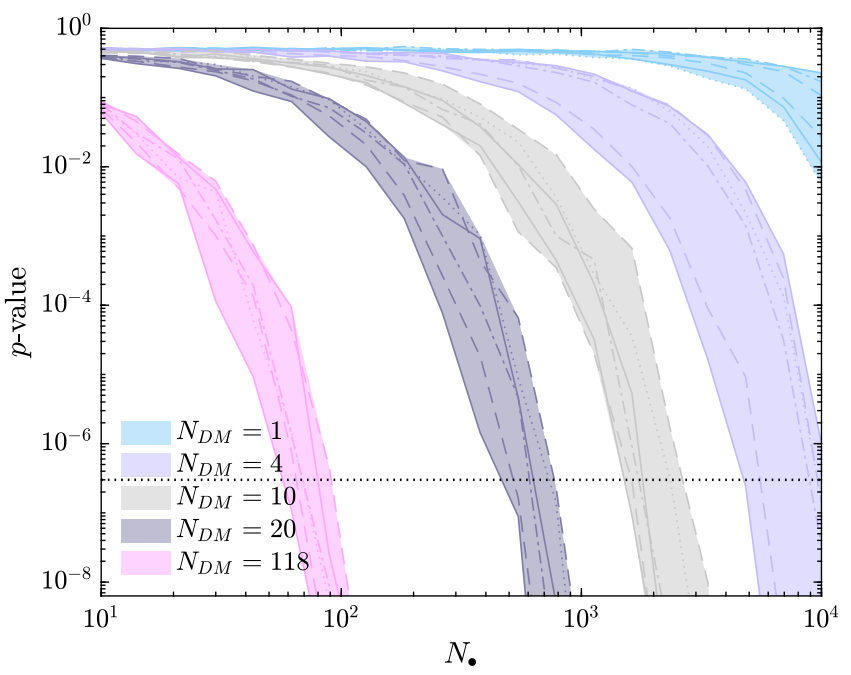

FIG. 3. Kolmogorov-Smirnov $p$-values obtained from missing transverse momentum observations, due to the evaporation of microscopic BHs into dark sector particles, compared with the case where only SM particles exist. We assume the number of extra dimensions is known. Different light bands represent dark sectors with 1 (blue, top), 4 (purple), 10 (light grey) 20 (dark grey) and 118 (pink, bottom-left) new particles. Within each band, thin lines are the specific cases of 1 (leftmost), 2, 3, 4, 5 and 6 (rightmost, least constrained) large extra dimensions. The horizontal dotted line depicts the $5 \sigma$ significance threshold. 
improves prospects significantly: if the DS contains as many d.o.f. as the SM, fewer than 100 events can robustly establish its existence.

As a consistency check, we also performed a binned chisquare test. The significance thus obtained is similar in behavior to the KS results, though slightly weaker, owing to the KS method's larger sensitivity to deviations from the expected distribution. These results are available in the Supplemental Material [43]. We furthermore checked that the change in $\not \not^{\perp}$ distributions follows the same trend when angular momentum is included (also shown in the Supplemental Material [43]). We did not perform a statistical analysis in this case, because the lack of a bulk graviton emission model for rotating BHs leads to considerable changes in the missing momentum statistics.

Detecting such an extended dark sector thus requires hundreds (for a large DS) to $\sim 10^{4}$ observations of $\mathrm{BH}$ decay. We find that the cross section to produce BHs for $M_{\star}=10 \mathrm{TeV}$ with a c.m. beam energy of $100 \mathrm{TeV}$ ranges from 13.5 to 640 picobarns. Producing $N$. BH events requires an integrated luminosity that scales as

$$
\frac{L}{6 \times 10^{36} \mathrm{~cm}^{-2}} \simeq \frac{N \cdot}{1000} 10^{0.061(n-6)^{2}},
$$

or between $10^{38} \mathrm{~cm}^{-2}(n=2$ extra dimensions) and $6 \times 10^{36} \mathrm{~cm}^{-2} \quad(n=6)$ to produce $\sim 1000$ BHs. Estimates of the FCC-hh requirements and capacity put its luminosity at 0.2 to 2 inverse attabarns $\left(10^{42} \mathrm{~cm}^{-2}\right)$ [44]. If LEDs are large enough, a sufficient quantity will be produced in the next generation of hadron colliders to unveil the nature of the DS, as long as its constituent particles are lighter than the BHs being produced.

Discussion and conclusions. - If the fundamental scale of quantum gravity indeed lies just above the reach of the LHC, the discovery of large extra dimensions via the creation of BHs opens up a trove of new physics possibilities. We have illustrated this by quantifying the prospects of discovering a new sequestered sector of particle physics. If dark matter couples only via gravity, the $\mathrm{BH}$ portal provides one of the only ways of individually detecting DM particles. Because the collider signatures become significantly different with many new d.o.f., we note that existing LHC limits may need to be recomputed; the Planck scale may indeed be closer at hand than previously thought.

We have focused on the prospects of simply detecting an extended dark sector. However, many phenomenological questions remain open, including the full effect of different LED properties, such as brane tension and splitting, compactification scheme, and geometry. We have also assumed that the full LED properties were under control, though we do expect some degeneracies between, e.g., bulk graviton and DM production. Full spectroscopy of the dark sector remains a difficult task. If the total missing energy and momentum can be obtained, e.g., at an $e^{+} e^{-}$collider, it is possible to reconstruct the mass and spin statistics of invisible primary evaporation products. Nonetheless, if LEDs are present at the $\sim 10 \mathrm{TeV}$ scale, they become the only guaranteed probe of any new dark sector below $M \sim M_{\star}$.

We thank Shivesh Mandalia for his help, as well as Joe Bramante and Carlos Tamarit for valuable input. We thank the anonymous referees for insightful comments and suggestions. This work was supported by the Arthur B. McDonald Canadian Astroparticle Physics Research Institute. Computations were performed at the Centre for Advanced Computing, and supported in part by the Canada Foundation for Innovation. Research at Perimeter Institute is supported by the Government of Canada through the Department of Innovation, Science, and Economic Development, and by the Province of Ontario through the Ministry of Economic Development, Job Creation and Trade.

*ningqiang.song@queensu.ca

†aaron.vincent@queensu.ca

[1] N. Aghanim et al. (Planck Collaboration), Planck 2018 results. VI. Cosmological parameters, arXiv:1807.06209.

[2] R. J. Scherrer and M.S. Turner, On the relic, cosmic abundance of stable weakly interacting massive particles, Phys. Rev. D 33, 1585 (1986); Erratum, Phys. Rev. D 34, 3263 (1986).

[3] D. E. Kaplan, M. A. Luty, and K. M. Zurek, Asymmetric dark matter, Phys. Rev. D 79, 115016 (2009).

[4] N. Arkani-Hamed, S. Dimopoulos, and G. R. Dvali, The hierarchy problem and new dimensions at a millimeter, Phys. Lett. B 429, 263 (1998).

[5] I. Antoniadis, N. Arkani-Hamed, S. Dimopoulos, and G. R. Dvali, New dimensions at a millimeter to a Fermi and superstrings at a TeV, Phys. Lett. B 436, 257 (1998).

[6] N. Arkani-Hamed, S. Dimopoulos, and G. R. Dvali, Phenomenology, astrophysics and cosmology of theories with submillimeter dimensions and $\mathrm{TeV}$ scale quantum gravity, Phys. Rev. D 59, 086004 (1999).

[7] P. C. Argyres, S. Dimopoulos, and J. March-Russell, Black holes and submillimeter dimensions, Phys. Lett. B 441, 96 (1998).

[8] L. Randall and R. Sundrum, A Large Mass Hierarchy from a Small Extra Dimension, Phys. Rev. Lett. 83, 3370 (1999).

[9] L. Randall and R. Sundrum, An Alternative to Compactification, Phys. Rev. Lett. 83, 4690 (1999).

[10] J. Murata and S. Tanaka, A review of short-range gravity experiments in the LHC era, Classical Quantum Gravity 32, 033001 (2015).

[11] C. Hanhart, J. A. Pons, D. R. Phillips, and S. Reddy, The likelihood of GODs' existence: Improving the SN1987a constraint on the size of large compact dimensions, Phys. Lett. B 509, 1 (2001).

[12] S. Hannestad and G. G. Raffelt, Supernova and neutron star limits on large extra dimensions reexamined, Phys. Rev. D 67, 125008 (2003); Erratum, Phys. Rev. D 69, 029901 (2004). 
[13] K. J. Mack and R. McNees, Bounds on extra dimensions from micro black holes in the context of the metastable Higgs vacuum, Phys. Rev. D 99, 063001 (2019).

[14] A. M. Sirunyan et al. (CMS Collaboration), Search for black holes and sphalerons in high-multiplicity final states in proton-proton collisions at $\sqrt{s}=13 \mathrm{TeV}$, J. High Energy Phys. 11 (2018) 042.

[15] A. M. Sirunyan et al. (CMS Collaboration), Search for new physics in dijet angular distributions using proton-proton collisions at $\sqrt{s}=13 \mathrm{TeV}$ and constraints on dark matter and other models, Eur. Phys. J. C 78, 789 (2018).

[16] K. Thorne, Black Holes \& Time Warps: Einstein's Outrageous Legacy (Commonwealth Fund Book Program) (W.W. Norton \& Company, New York, 1995).

[17] T. Banks and W. Fischler, A model for high-energy scattering in quantum gravity, arXiv:hep-th/9906038.

[18] S. Dimopoulos and G. L. Landsberg, Black Holes at the LHC, Phys. Rev. Lett. 87, 161602 (2001).

[19] S. B. Giddings and S. D. Thomas, High-energy colliders as black hole factories: The end of short distance physics, Phys. Rev. D 65, 056010 (2002).

[20] A. Chamblin, F. Cooper, and G. C. Nayak, SUSY production from TeV scale blackhole at CERN LHC, Phys. Rev. D 70, 075018 (2004).

[21] C. M. Harris, Physics beyond the standard model: Exotic leptons and black holes at future colliders, Ph.D. thesis, Cambridge University, Cambridge, England, 2004.

[22] M. Cavaglia, R. Godang, L. Cremaldi, and D. Summers, Catfish: A Monte Carlo simulator for black holes at the LHC, Comput. Phys. Commun. 177, 506 (2007).

[23] G. L. Alberghi, R. Casadio, and A. Tronconi, Quantum gravity effects in black holes at the LHC, J. Phys. G 34, 767 (2007).

[24] M. Cavaglia, R. Godang, L. M. Cremaldi, and D. J. Summers, Signatures of black holes at the LHC, J. High Energy Phys. 06 (2007) 055.

[25] X. Calmet, W. Gong, and S. D. H. Hsu, Colorful quantum black holes at the LHC, Phys. Lett. B 668, 20 (2008).

[26] G. C. Nayak, Dark matter production at LHC from black hole remnants, Phys. Part. Nucl. Lett. 8, 337 (2011).

[27] D. M. Gingrich, Quantum black holes with charge, colour, and spin at the LHC, J. Phys. G 37, 105008 (2010).

[28] D. M. Gingrich, Monte Carlo event generator for black hole production and decay in proton-proton collisions, Comput. Phys. Commun. 181, 1917 (2010).

[29] G. Landsberg, Black holes at the large hadron collider, Fundam. Theor. Phys. 178, 267 (2015).

[30] S. W. Hawking, Particle creation by black holes, Commun. Math. Phys. 43, 199 (1975).

[31] M. Benedikt and F. Zimmermann, Future circular colliders, Proc. Int. Sch. Phys. Fermi 194, 73 (2016).
[32] J. Tang et al., Concept for a future super proton-proton collider, arXiv:1507.03224.

[33] S. Chatrchyan et al. (CMS Collaboration), Search for microscopic black holes in $p p$ collisions at $\sqrt{s}=8 \mathrm{TeV}$, J. High Energy Phys. 07 (2013) 178.

[34] G. Aad et al. (ATLAS Collaboration), Search for microscopic black holes in a like-sign dimuon final state using large track multiplicity with the ATLAS detector, Phys. Rev. D 88, 072001 (2013).

[35] D.-C. Dai, G. Starkman, D. Stojkovic, C. Issever, E. Rizvi, and J. Tseng, BlackMax: A black-hole event generator with rotation, recoil, split branes, and brane tension, Phys. Rev. D 77, 076007 (2008).

[36] H. Yoshino and V. S. Rychkov, Improved analysis of black hole formation in high-energy particle collisions, Phys. Rev. D 71, 104028 (2005); Erratum, Phys. Rev. D 77, 089905 (2008).

[37] D.-C. Dai, C. Issever, E. Rizvi, G. Starkman, D. Stojkovic, and J. Tseng, Manual of BlackMax. A black-hole event generator with rotation, recoil, split branes, and brane tension. Version 2.02, Comput. Phys. Commun. 236, 285 (2019).

[38] T. Sjstrand, S. Ask, J. R. Christiansen, R. Corke, N. Desai, P. Ilten, S. Mrenna, S. Prestel, C. O. Rasmussen, and P.Z. Skands, An introduction to PYTHIA 8.2, Comput. Phys. Commun. 191, 159 (2015).

[39] S. Dulat, T.-J. Hou, J. Gao, M. Guzzi, J. Huston, P. Nadolsky, J. Pumplin, C. Schmidt, D. Stump, and C.-P. Yuan, New parton distribution functions from a global analysis of quantum chromodynamics, Phys. Rev. D 93, 033006 (2016).

[40] A. Buckley, J. Ferrando, S. Lloyd, K. Nordström, B. Page, M. Rüfenacht, M. Schönherr, and G. Watt, LHAPDF6: Parton density access in the LHC precision era, Eur. Phys. J. C 75, 132 (2015).

[41] D. Stojkovic, Distinguishing Between the Small ADD and RS Black Holes in Accelerators, Phys. Rev. Lett. 94, 011603 (2005).

[42] R. Foot, H. Lew, and R. R. Volkas, A model with fundamental improper space-time symmetries, Phys. Lett. B 272, 67 (1991).

[43] See Supplemental Material at http://link.aps.org/ supplemental/10.1103/PhysRevLett.124.051801 for rotating black hole cases and binned likelihood.

[44] F. Zimmermann, M. Benedikt, X. Buffat, and D. Schulte, Luminosity targets for FCC-hh, in Proceedings, 7th International Particle Accelerator Conference (IPAC 2016): Busan, Korea, 2016 (JACoW, Geneva, Switzerland, 2016), http://accelconf.web.cern.ch/AccelConf/ipac2016/ doi/JACoW-IPAC2016-TUPMW037.html. 